\title{
Preferential segregation of Pd atoms in the Ag-Pd bimetallic cluster: Density functional theory and molecular dynamics simulation
}

\author{
Hyun You Kim, Hyoung Gyu Kim, Ji Hoon Ryu, and Hyuck Mo Lee* \\ Department of Materials Science and Engineering, Korea Advanced Institute of Science and Technology, \\ Gusung-dong 373-1, Yusung-gu, Daejeon 305-701, Korea
}

(Received 9 February 2007; published 21 June 2007)

\begin{abstract}
Classical molecular dynamics simulations of the coalescence between an Ag cluster composed of 135 atoms and a Pd cluster of 16 atoms were performed at $500 \mathrm{~K}$. All Pd atoms penetrated into the $\mathrm{Ag}$ cluster and preferentially segregated at the subsurface layer. The density functional theory calculations revealed that the center site is the most stable position for Pd atoms. However, the energy barrier for further penetration of Pd atoms located at the subsurface was too high to overcome, and thus a metastable structure with Pd atoms segregated at the subsurface did not evolve.
\end{abstract}

DOI: 10.1103/PhysRevB.75.212105

PACS number(s): 61.46.Df, 71.15.Mb

It is well known that the bimetallic clusters composed of two different elements show unique optical and catalytic properties compared to single element clusters. ${ }^{1-4}$ Thus, investigation of the overall morphology and structural stability of bimetallic clusters has been one of the most important requirements for the practical application of bimetallic clusters. $^{5-7}$ In general, the composite structure of bimetallic clusters such as core-shell or onionlike structures can be observed depending on the cluster system. ${ }^{6-10}$ It has been reported and believed that the significant difference in the atomic size and surface energy causes preferential surface segregation of the component having relatively large size and low surface energy, and gives rise to specific cluster morphology. ${ }^{9-11}$

Several computational methods such as genetic algorithms and Monte Carlo methods have been applied to investigate the globally stable structure of bimetallic clusters. ${ }^{6-8}$ It was confirmed that the core-shell structure is most stable in several bimetallic systems. In a recent report, Baletto et al. showed that a specific structure of a bimetallic cluster with subsurface segregated solute atoms can be found in Ag-based bimetallic cluster systems. ${ }^{8}$ They assumed that this is a metastable state, because all solute atoms penetrated into the cluster center at higher temperature.

In our previous molecular dynamics (MD) study of the coalescence between 135-Ag and 16-Pd clusters at $300 \mathrm{~K}$, we observed penetration of $\mathrm{Pd}$ atoms into the $\mathrm{Ag}$ cluster and the formation of local fivefolded icosahedron (Ih) structures. ${ }^{5}$ However, some Pd atoms remained at the cluster surface, and thus did not segregate into the cluster core. As a result, the core-shell cluster structure was not observed, which was explained by the strong mixing nature between $\mathrm{Ag}$ and $\mathrm{Pd}$ atoms and, more importantly, low kinetic energy. Thus, in the present work, we study the coalescence between 135-Ag and 16 -Pd clusters at a somewhat high temperature of $500 \mathrm{~K}$ and compare the results with previous findings. We discuss the structural stability of the Ag-Pd cluster on the basis of density functional theory (DFT) and MD.

The MD simulation was carried out at $500 \mathrm{~K}$ under an NVT ensemble using a quantum Sutton-Chen (QSC) potential. ${ }^{12}$ The relevant potential parameters of $\mathrm{Ag}$ and $\mathrm{Pd}$ together with detailed MD simulation conditions were de- scribed in our previous study. ${ }^{5}$ An Ag cluster composed of 135 atoms and a Pd cluster of 16 atoms were individually prepared and fully relaxed at $500 \mathrm{~K}$. They were later placed in a large simulation box $(500 \AA \times 500 \AA \times 500 \AA)$ and collided with each other. The MD simulation of the collision was carried out for 1000 ps (five million time steps).

The DFT calculations were performed using the $\mathrm{DMol}^{3}$ package in the Materials Studio. ${ }^{13}$ The Kohn-Sham equation was expanded in a local atomic orbital basis. The unrestricted Hartree-Fock (UHF) method with a double-numeric quality basis set with polarization functions (DNP) was applied. The effective core potential was applied to treat core electrons of heavy $\mathrm{Ag}$ and $\mathrm{Pd}$ atoms. ${ }^{14}$ The exchangecorrelation functional in the generalized gradient approximation (GGA) was parametrized by the Perdew and Wang scheme. ${ }^{15}$ A Fermi smearing of 0.002 hartree (1 hartree $=27.2114 \mathrm{eV}$ ) was used.

The transition state calculations were performed using synchronous transit methods, the linear synchronous transit (LST), and quadratic synchronous transit (QST), ${ }^{16}$ in combination with the conjugate gradient (CG) minimization algorithm for subsequent refinement. ${ }^{17}$ In addition, the minimum energy path between the initial structure, transition structure, and final structure was investigated using the nudged elastic band (NEB) theory. ${ }^{18}$

Figure 1 summarizes the overall structure of the Ag-Pd cluster after $1 \mathrm{~ns}$ of MD simulation. Unlike at $300 \mathrm{~K}$, all $\mathrm{Pd}$ atoms penetrated into the cluster, as seen in Fig. 1(a). This indicates that the average kinetic energy of $\mathrm{Pd}$ atoms was sufficient to overcome the energy barrier of penetration at $500 \mathrm{~K}$. Almost (14 of 16) all of the penetrated Pd atoms preferentially segregated at the subsurface layer (one layer below the surface) of the cluster, as shown in Fig. 1(b), which is in partial agreement with the work of Baletto et al. ${ }^{8}$ They obtained a three-shell onionlike bimetallic nanocluster, and explained this abnormal cluster structure by referring to the most favorable site of impurities in an energetic manner. The penetrated $\mathrm{Pd}$ atoms induced surface disordering of the $\mathrm{Ag}$ cluster and the disordered Ag atoms at the surface were locally rearranged into Ih structures, Fig. 1(c).

Interestingly, in spite of relatively high simulation temperature, most Pd atoms (14 of 16) preferred to segregate at 

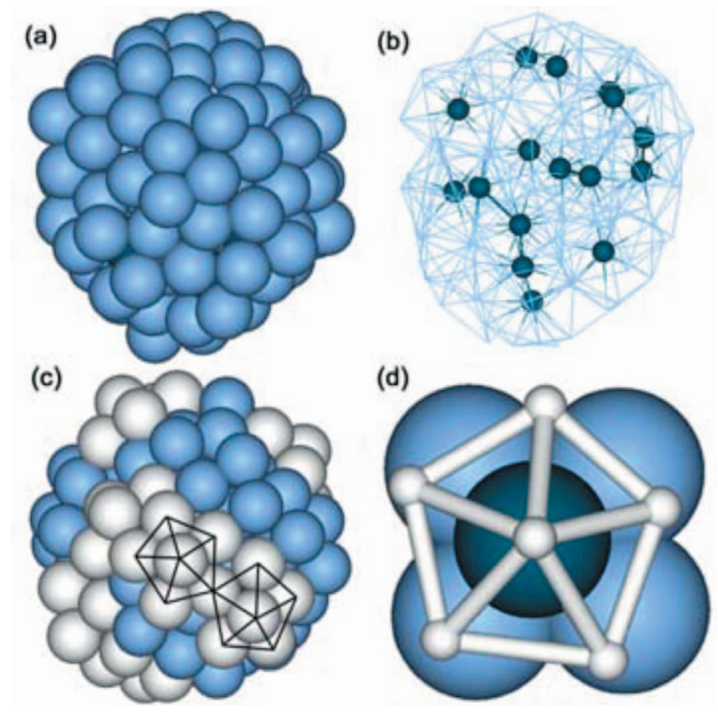

FIG. 1. (Color online) An Ag-Pd cluster after 1 ns of MD simulation at $500 \mathrm{~K}$ : (a) Overall morphology of the cluster surface, (b) distribution of Pd atoms delineating $14 \mathrm{Pd}$ atoms out of 16 preferentially segregated at the subsurface layer, (c) distribution of densely packed Ih structures on the surface. Pentagonal lines denote individual Ih structure at the surface. (d) Perspective view of one Ih showing one Pd atom embedded in Ih, so-called Ih-Pd. Ag atoms are colored light blue and Pd atoms colored dark green. White colored atoms represent the $\mathrm{Ag}$ atoms involved in the Ih structure (the same coloring is used throughout the figures).

the subsurface layer rather than coalescing in the cluster core, thus making a core-shell structure. In addition, penetrated $\mathrm{Pd}$ atoms were combined with the Ih structure at the surface, giving rise to Ih-Pd structures. One Ih-Pd is composed of six $\mathrm{Ag}$ atoms and one $\mathrm{Pd}$ atom embedded at the center, Fig. 1(d). The surface of the collided Ag-Pd cluster was covered with densely packed Ih-Pd and it persisted during the MD simulation. As a result, about two-thirds of the total surface area of the Ag-Pd bimetallic cluster was covered with Ih-Pd packages [Figs. 1(c) and 1(d)]. Once the Ih-Pd structure appeared, it maintained its morphology during the entire simulation period. Fourteen among a total of $16 \mathrm{Pd}$ atoms were observed at the subsurface layer and 13 of them were involved in the Ih-Pd packages.

The local Ih-Pd structure was also observed at $300 \mathrm{~K}$, though not as frequently. ${ }^{5}$ At $300 \mathrm{~K}$, the Ih structure at the surface was expanded, developing a (111) plane. However, in this study at $500 \mathrm{~K}$, all $\mathrm{Pd}$ atoms rapidly penetrated the cluster and many Ih structures were densely distributed on the cluster surface (61 surface atoms of the total 98 atoms that constitute the Ag-Pd cluster surface were involved in constituting Ih). This explains why an expanded (111) plane did not develop at $500 \mathrm{~K}$.

Nam et al. reported that in studying the Au nanocluster, the liquid-to-Ih transition was initiated and affected by early transformation of the cluster surface layer during the cooling process, because the surface energy reduction is more dominant than the cluster internal energy reduction. ${ }^{19}$ The current study showed that the internal atomistic configuration of the bimetallic cluster (regarding the location of Pd atoms) could

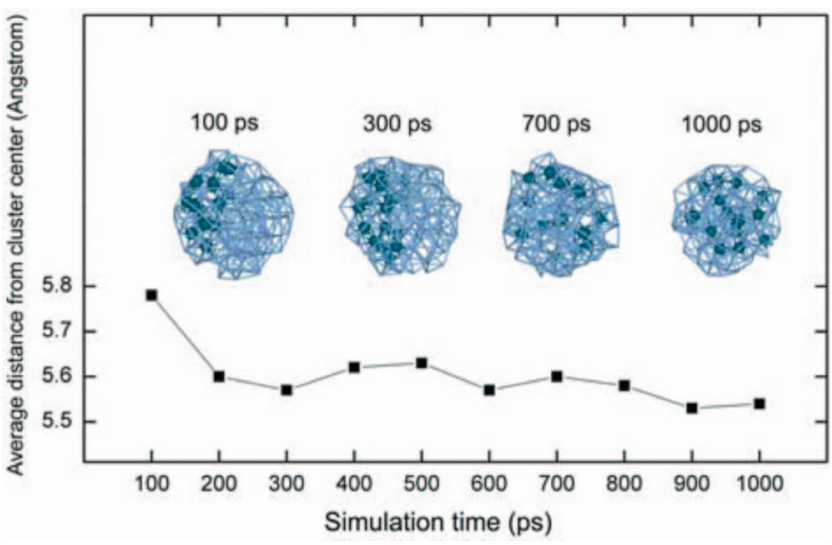

FIG. 2. (Color online) Variation of average distance between the $\mathrm{Pd}$ atoms and the cluster center with simulation time. It shows movement of Pd atoms along the subsurface layer of the Ag-Pd cluster. The fluctuation in average distance between $\mathrm{Pd}$ atoms and cluster center has significantly diminished after 200 ps, if any.

also be affected by the surface morphology of the cluster. Although our MD simulation was performed at constant temperature, penetration of $\mathrm{Pd}$ atoms induced surface disordering and formation of Ih structures, followed by rearrangement of penetrated $\mathrm{Pd}$ atoms.

The Pd atoms eventually moved into specific positions below the Ih structures [Fig. 1(d)], giving rise to Ih-Pd packages. It is highly likely that the relative weak tendency of formation of a core-shell structure in the Ag-Pd system delayed further penetration of $\mathrm{Pd}$ atoms into the cluster center. This accounts for why the Pd atoms did not segregate at the cluster center in an early stage of the MD simulation.

It is notable that the penetrated $\mathrm{Pd}$ atoms mostly remained and moved on the near subsurface layer of the Ag-Pd cluster during the entire simulation period. According to Fig. 2 that shows this tendency, the average distance between the cluster center and Pd atoms did not change significantly after 200 ps and the Pd atoms moved along the subsurface layer of the cluster. Finally, Pd atoms moved into specific positions below the Ih structures.

In order to understand why the Ih-Pd structures are maintained stable, we modeled one Ih-Pd structure with a 55-Ih cluster composed of $54 \mathrm{Ag}$ atoms and one $\mathrm{Pd}$ atom. There are three atomic positions along the cluster axis in the 55-Ih cluster from vertex to center: vertex, subsurface, and center sites. With respect to the Pd location, DFT calculations on the total energy of the cluster were performed. Initially, each cluster structure was geometrically optimized before total energy calculations. The total energy of the 55-Ih decreased by -0.55 and $-0.27 \mathrm{eV}$, respectively, as the $\mathrm{Pd}$ atom moved from the vertex to subsurface site and from the subsurface to the center. This reflects a general tendency observed in the bimetallic system. ${ }^{11}$ The Gibbs free energy difference calculated between two 55-Ih structures, one with $\mathrm{Pd}$ at the subsurface and another with $\mathrm{Pd}$ at the center, showed that it is smaller than zero and decreases monotonically as a function of temperature. This also implies that the cluster center is the most stable site for Pd. However, the current study revealed that the Pd atoms preferentially segregated at the subsurface 


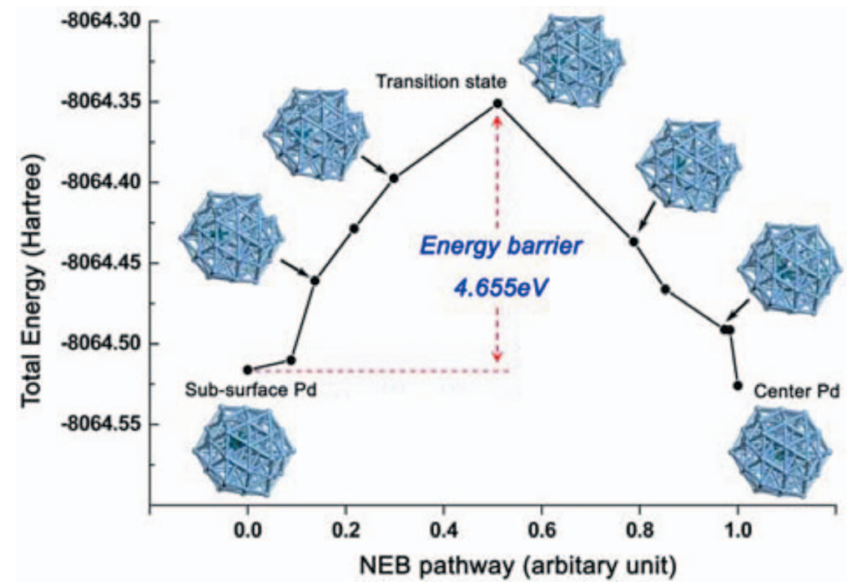

FIG. 3. (Color online) Schematic NEB pathway between two 55-Ih structures, one with Pd at the subsurface and another with Pd at the center. Each snapshot shows evolution of the cluster structure with respect to movement of a Pd atom.

sites and remained there. Thus, we assumed that once an Ih-Pd package is formed, the energy barrier for further penetration of Pd to the center is large enough to hinder movement of $\mathrm{Pd}$ and to offset the driving force for minimization of the total energy.

To test this assumption, the LST and/or QST and CG methods were applied to determine the energy barrier for penetration of Pd from the subsurface to the center and the transient state structure. The minimum energy path was estimated by the NEB method based on the LST and/or QST and $\mathrm{CG}$ results. Figure 3 shows the structures at the transition state and the energy barrier for Pd from the subsurface to the center. The estimated minimum energy path is also shown. The energy barrier was calculated as $4.655 \mathrm{eV}$. This is much larger than the kinetic energy averaged for individual atoms at $500 \mathrm{~K}, 0.065 \mathrm{eV}$. Thus, the large energy barrier prevents further penetration of $\mathrm{Pd}$ atoms to the cluster center and, as a result, the Ih structures with Pd at the subsurface were stabilized, although at a metastable level.

Baletto et al. noted that in deposition of $200 \mathrm{Pd}$ atoms over a cluster of 201 or $147 \mathrm{Ag}$ atoms the impurity (Pd) energy in the Ag cluster was very similar between the subsurface and the center sites. ${ }^{8}$ In the case of the Ih-structured Ag cluster, the center site was as favorable as the subsurface site due to the small size mismatch between $\mathrm{Ag}$ and $\mathrm{Pd}$. In this study, we only employed a 55-Ih, and thus this result cannot be directly compared with their result. ${ }^{8}$ Nonetheless, our DFT total energy calculations of the 55-Ih cluster showed that there is reliable evidence of an energy difference between two 55-Ih structures, one with Pd at the subsurface and another with $\mathrm{Pd}$ at the center. As mentioned earlier, the penetrated $\mathrm{Pd}$ atoms moved on the subsurface layer during MD simulations. However, it is not yet clear why the Pd atoms moved into specific positions, as shown in Fig. 1(d). This aspect is currently being investigated.

Recent studies on the Pd-Pt bimetallic cluster system reported the appearance of an onionlike cluster and site pref- erence of the Pt solute atom. ${ }^{6,7}$ Lloyd et al. showed that $\mathrm{Pt}$ solute atoms preferred the subsurface position in the $\mathrm{Pd}-\mathrm{Pt}$ 55-Ih cluster. ${ }^{7}$ Referring to their report and considering our result, it is likely that the nature of distribution of solute atoms in the bimetallic cluster is different depending on the kind of cluster systems.

Another point that must be considered is that the energy barrier estimated by the synchronous transit method is an upper limit to the barrier height and the NEB minimum energy path was also estimated based on the LST, QST, and CG results. It is possible that there may be other pathways and the corresponding energy barriers could be lowered. The large gap between the calculated energy barrier and the average kinetic energy of individual atoms $(4.59 \mathrm{eV})$ suggests that even if there is a slight overestimation in our energy barrier value, it does not seriously affect the overall results obtained in this study. The estimated energy barrier was based on the ideal 55-Ih cluster, and thus it may be lowered by variation of local surface structures or the internal atomic configuration of the real cluster. This can make the $A-B-A$ to $A-B$ transition possible, as presented in the work of Baletto et $a .^{8}$

In general, the catalytic properties of metal surface and clusters are very sensitive to their composition and atomic distribution. ${ }^{20}$ It was asserted that the core-shell structure is most stable in the Ag-Pd bimetallic cluster. ${ }^{10}$ However, our results show that the metastable structure may be obtained and maintains its stability under certain specific conditions. The appearance of Ih and Ih-Pd at the surface must have exerted a significant effect on atomic distribution of the Ag-Pd cluster. Thus, it is highly likely that the catalytic properties of the Ag-Pd cluster may be different. As a result, our study suggests that the kinetic aspects such as the energy barrier between metastable and most stable structures or evolution of the cluster surface and related solute distribution should be carefully considered for the practical application of bimetallic clusters.

In summary, we showed that Pd atoms preferentially segregated at the subsurface layer of the Ag-Pd bimetallic cluster. After collision between Ag and Pd clusters, two-thirds of the surface Ag atoms were rapidly rearranged to Ih structures and $\mathrm{Pd}$ atoms moved into specific positions below the Ih thus, making Ih-Pd packages. It is found that the formation of the Ih-Pd structure (equal to preferential segregation of Pd atoms) and its stability were strongly affected by the surface morphology of the cluster (existence of Ih structures at the surface after collision). The local Ih-Pd structure is metastable and the high-energy barrier hinders movement of Pd atoms to more stable sites of the cluster center. This explains why the bimetallic cluster structure with subsurface located $\mathrm{Pd}$ atoms was preferred to the core-shell structure in this work.

Many discussions with Sang Soo Han at the Materials and Processing Simulation Center of CALTECH and Sung Hoon Lee at the Department of Materials Science and Engineering of the Pennsylvania State University are greatly appreciated. 
*Corresponding author. FAX: +82-42-869-3310.

hmlee@kaist.ac.kr

${ }^{1}$ M. Mavrikakis, B. Hammer, and J. K. Norskov, Phys. Rev. Lett. 81, 2819 (1998).

${ }^{2}$ Y. Mizukoshi, T. Fujimoto, Y. Nagata, R. Oshima, and T. Maeda, J. Phys. Chem. B 104, 6028 (2000).

${ }^{3}$ A. P. Alivisatos, J. Phys. Chem. 100, 13226 (1996).

${ }^{4}$ J. H. Sinfelt, Bimetallic Catalysts-Discoveries, Concepts, and Applications (Wiley, NewYork, 1983).

${ }^{5}$ H. Y. Kim, S. H. Lee, H. G. Kim, J. H. Ryu, and H. M. Lee, Mater. Trans., JIM 48, 455 (2007).

${ }^{6}$ D. Cheng, W. Wang, and S. Huang, J. Phys. Chem. B 110, 16193 (2006).

${ }^{7}$ L. D. Lloyd, R. L. Johnston, S. Salhi, and N. T. Wilson, J. Mater. Chem. 14, 1691 (2004).

${ }^{8}$ F. Baletto, C. Mottet, and R. Ferrando, Phys. Rev. Lett. 90, 135504 (2003).

${ }^{9}$ A. Rapallo, G. Rossi, R. Ferrando, A. Fortunelli, B. C. Curley, L. D. Lloyd, G. M. Tarbuck, and R. L. Johnston, J. Chem. Phys. 122, 194308 (2005).

${ }^{10}$ G. Rossi, R. Ferrando, A. Rapallo, A. Fortunelli, B. C. Curley, L. D. Lloyd, and R. L. Johnston, J. Chem. Phys. 122, 194309 (2005).

${ }^{11}$ O. M. Løvvik, Surf. Sci. 583, 100 (2005); A. V. Ruban, H. L. Skriver, and J. K. Norskov, Phys. Rev. B 59, 15990 (1999); F. Baletto, C. Mottet, and R. Ferrando, ibid. 66, 155420 (2002).

${ }^{12}$ T. Cagin, Y. Kimura, Y. Qi, H. Li, H. Ikeda, W. L. Johnson, and
W. A. Goddard III, in Proceedings of the Materials Research Society Symposia, edited by W. L. Johnson, C. T. Liu, and A. Inoue (Materials Research Society, Pittsburgh, 1999), p. 43; Y. Qi, T. Cagin, Y. Kimura, and W. A. Goddard III, J. Comput.Aided Mater. Des. 8, 233 (2001).

${ }^{13}$ B. Delly, J. Chem. Phys. 92, 508 (1990); 113, 7756 (2000); J. Phys. Chem. 100, 6107 (1996).

${ }^{14}$ M. Dolg, U. Wedig, H. Stoll, and H. Preuss, J. Chem. Phys. 86, 866 (1987); A. Bergner, M. Dolg, W. Kuechle, H. Stoll, and H. Preuss, Mol. Phys. 80, 1431 (1993).

${ }^{15}$ J. P. Perdew and Y. Wang, Phys. Rev. B 45, 13244 (1992).

${ }^{16}$ T. A. Halgen and W. A. Lipscomb, Chem. Phys. Lett. 49, 225 (1977); N. Govind, M. Petersen, G. Fitzgerald, D. King-Smith, and J. Andzelm, Comput. Mater. Sci. 28, 250 (2003).

${ }^{17}$ S. Bell and J. S. Crighton, J. Chem. Phys. 80, 2464 (1984).

${ }^{18}$ G. Henkelman and H. Jonsson, J. Chem. Phys. 113, 9978 (2000).

${ }^{19}$ H.-S. Nam, N. M. Hwang, B. D. Yu, and J.-K. Yoon, Phys. Rev. Lett. 89, 275502 (2002).

${ }^{20}$ J. Greeley and M. Mavrikakis, Nat. Mater. 3, 810 (2004); T. Jacob and W. A. Goddard III, J. Phys. Chem. B 108, 8311 (2004); Y. Xu, A. V. Ruban, and M. Mavrikakis, J. Am. Chem. Soc. 126, 4717 (2004); J. Zhang, M. B. Vukmirovic, K. Sasaki, A. U. Nilekar, M. Mavrikakis, and R. R. Adzic, ibid. 127, 12480 (2005); J. Zhang, M. B. Vukmirovic, Y. Xu, M. Mavrikakis, and R. R. Adzic, Angew. Chem., Int. Ed. 44, 2132 (2005); Y. Okamoto, Chem. Phys. Lett. 395, 279 (2004); 429, 209 (2006). 